\title{
Ferromagnetic Metals in High Magnetic Fields
}

\author{
A. J. Freeman, N. A. Blum, S. Foner, R. B. Frankel, and E. J. McNiff, JR. \\ National Magnet Laboratory, ${ }^{*}$ Massachusetts Institute of Technology, Cambridge, Massachusetts
}

\begin{abstract}
High-field susceptibility results are presented for Mössbauer measurements on iron at low temperatures in fields up to $135 \mathrm{kOe}$ and magnetization measurements on iron and nickel single crystals in fields up to 148 $\mathrm{kOe}$ with vibrating sample magnetometers. The Mössbauer measurements give the hyperfine field $H_{n}=$ $H_{\text {int }}-\left(H_{0}-H_{\mathrm{DM}}\right)+\Delta H$, where $H_{\text {int }}$ is the hyperfine field at zero applied field, $H_{0}$ is the applied field, $H_{\mathrm{DM}}$ is the demagnetizing field, and $\Delta H$ is a term which would reflect a change in the magnetization at high field, i.e., $\Delta H / H_{n}=\Delta M / M$. The Mössbauer results at $4.2^{\circ} \mathrm{K}$ are equivalent to $\chi=2 \pm 7 \times 10^{-5} \mathrm{emu} / \mathrm{cc}$. The magnetization experiments give $\chi \leq 4 \times 10^{-5}$ for iron and $\chi \leq 1.1 \times 10^{-5}$ for nickel at $4.2^{\circ} \mathrm{K}$. We discuss the various contributions to the total susceptibility $\chi$, give estimates of $\chi_{d}$ based on theoretical band calculations, and discuss various uncertainties in both the theoretical calculations and in values of the molecular field parameter which make detailed comparisons difficult. Finally, we compare with predictions based on Herring's phenomenological description of $x$ for ferromagnets.
\end{abstract}

\section{INTRODUCTION}

$\mathbf{T}$ $\mathrm{HE}$ change of magnetization with applied magnetic field is an important property of ferromagnets because it allows a comparison with the predictions of band, or collective-electron, theory ${ }^{1}$ and may have relevance in deciding between itinerant versus localized models of ferromagnetism. ${ }^{2}$ Wohlfarth ${ }^{1}$ has called attention to the fact that the collective-electron theory ${ }^{3}$ predicts a relative magnetization at absolute zero $\zeta_{0}$ which may be less than one, depending on details of the density of states of the metal, $N(E)$, and the strength of the Weiss molecular field. The application of an intense external magnetic field should cause, at very low temperatures, an increase in $\zeta_{0}$ if $\zeta_{0}$ is indeed $<1$. Such a situation is, however, incompatible with theories based on localized atomic moments which require $^{1,2} \zeta_{0} \simeq 1$ at $T=0^{\circ} \mathrm{K}$.

In collective electron theory, the high-field band susceptibility $\chi_{d}$ at $T=0^{\circ} \mathrm{K}$ is given by

$$
n \mu^{2} / \chi_{d}=\frac{1}{4} n\left\{\left[1 / N\left(\epsilon_{F \uparrow}\right)\right]+\left[1 / N\left(\epsilon_{F \downarrow}\right)\right]\right\}-k \theta^{\prime},
$$

if one ignores other contributions (discussed later). This result assumes that the total energy is the sum of single-particle energies plus an added exchange energy. In Eq. (1), $n$ is the total number of electrons, $k \theta^{\prime}$ is the molecular field represented by a characteristic temperature $\theta^{\prime}$, and $N\left(\epsilon_{F \uparrow}\right)$ and $N\left(\epsilon_{F_{\ddagger}}\right)$ are the spin-up and spin-down densities of states at the Fermi energy, respectively. It clear that if $\zeta_{0}=1, \chi_{d}=0$, since $N\left(\epsilon_{F \uparrow}\right)$ or $N\left(\epsilon_{\mathbb{b}}\right)=0$.

\section{MAGNETIZATION STUDIES}

The smallest previous limits of the high-field susceptibility in $\mathrm{Fe}$ and $\mathrm{Ni}$ were established by Kapitza. ${ }^{4}$

\footnotetext{
* Work supported by the U.S. Air Force Office of Scientific Research.

1 E. P. Wohlfarth, Phys. Letters 3, 17 (1962).

2 C. Herring, in Magnetism, edited by H. Suhl and G. T. Rado (Academic Press Inc., New York, to be published), Vol. IV.

${ }^{3}$ E. C. Stoner, Phys. Soc. Progr. Phys. 11, 43 (1948); Proc. Roy. Soc. (London) A165, 372 (1938).

4 P. Kapitza, Proc. Roy. Soc. (London) A131, 243 (1931); see also W. E. Henry, Phys. Rev. 99, A668 (1955).
}

For pulsed field changes of about $250 \mathrm{kOe}$, he found that the change in the magnetization was less than $1 \%$ (the limits of his error).

It is important to note that absolute sensitivity or accuracy is not a major problem for these large moment measurements; rather, high relative differential sensitivity is required, and usually negligible systematic and instrumental corrections become dominant. We have combined the highest readily available fields with two magnetometers for these measurements. First, differential magnetic moments (DMM) were measured at $4.2^{\circ} \mathrm{K}$ up to $80 \mathrm{kOe}$ in superconducting magnets with a vibrating sample magnetometer $(\mathrm{VSM})^{5}$ modified for the low-temperature, axial-field configuration. Small single-crystal, spherical samples of $\mathrm{Fe}$ and $\mathrm{Ni}$ subjected to an applied field along the easy axes showed very small susceptibility over this field range. Second, DMM measurements were extended with a simplified VSM assembled for operation in the NML watercooled, high-field solenoids. Brieffy, this instrument is a low-frequency VSM of moderate sensitivity which combines flux integration, time averaging, and calibrated high-differential stability, and is specifically arranged to eliminate possible sample-positioning errors. ${ }^{6}$

The present measurements on $\mathrm{Fe}$ and $\mathrm{Ni}$ single crystals up to $148 \mathrm{kOe}$ with the latter instrument show high-field susceptibilities for $\chi_{\mathrm{Fe}} \leq 4 \times 10^{-5}$ and $\chi_{\mathrm{Ni}} \leq 1.1 \times 10^{-5}$. The reproducibility of any data point over the entire field range had a maximum deviation of less than $0.05 \%$ to $0.1 \%$. The available large field range permitted the detection and elimination of small systematic errors. Our results are in agreement with earlier measurements of Kapitza ${ }^{4}$ and Henry ${ }^{4}$ and with the Mössbauer measurements described below. We expect that any remaining systematic errors are small compared to the quoted upper limits and that the actual values are very close to these limits.

\footnotetext{
${ }^{5} \mathrm{~S}$. Foner, Rev. Sci. Instr. 30, 548 (1959).

${ }^{6}$ Further details about this instrument will be described elsewhere. S. Foner and E. J. McNiff, Jr. (to be published).
} 


\section{MÖSSBAUER STUDIES}

The Mössbauer experiments were performed using a source of ${ }^{57} \mathrm{Co}$ in metallic iron together with an ${ }^{57} \mathrm{Fe}$ enriched metallic iron foil absorber. Both source and absorber were at $4.2^{\circ} \mathrm{K}$ and in an external longitudinal magnetic field of about $135 \mathrm{kOe}$, resulting in a three-line absorption spectrum. ${ }^{7}$ The splitting between the outer lines is proportional to the hyperfine field $H_{n}$ assuming $H_{n}$ to be the same in both source and absorber. This configuration results in a much higher counting rate than if an unsplit single-line source at zero external field were used; consequently the statistical error is reduced for a given available counting time. Consider $H_{n}=H_{\text {int }}+H_{\mathrm{DM}}+\Delta H-H_{0}$, with $H_{n}$ the observed hyperfine field, $H_{\text {int }}$ the hyperfine field at zero external field $\left(338 \mathrm{kOe}\right.$ at $\left.4.2^{\circ} \mathrm{K}\right),-\mathrm{H}_{0}$ the applied field, $H_{\mathrm{DM}}$ the demagnetizing field (21.8 $\mathrm{kOe}$ for a thin iron foil), ${ }^{8}$ and $\Delta H$ the discrepancy corresponding to a change in the magnetization. We assume strict proportionality between the hyperfine field and the magnetization. In terms of the volume susceptibility our result corresponds to $\chi=[\Delta H \times M] /\left[H_{n} \times\left(H_{0}-H_{\mathrm{DM}}\right)\right]$, where $M$ is the saturation magnetization in iron $(\sim 1.7 \mathrm{kG})$. Combining the results of three separate determinations of $\Delta H$, we obtain the results, $\Delta H=0.3 \pm 1.3 \mathrm{kOe}$, and $x=(2 \pm 7) \times 10^{-5} \mathrm{emu} / \mathrm{cc}$.

\section{DISCUSSION}

The magnetic susceptibility of a metal, based on a simplified tight binding model of two types of bands ( $d$ and $s$ ), may be written as $\chi=\chi_{s}+\chi_{d}+\chi_{v v}+\chi_{\text {diam }}$, where $\chi_{s}$ and $\chi_{d}$ are the spin paramagnetic contributions, $\chi_{\mathrm{vv}}$ is the paramagnetic contribution for partially filled degenerate bands (or Van Vleck temperatureindependent paramagnetism for metals), and $\chi_{\text {diam }}$ is the diamagnetic contribution. Wę wish to deduce information about the band structure of ferromagnetic metals from measurements of $\chi$ at low temperatures. To do this we must first estimate the other contributions to $\chi$. From measurements of $\chi$ in $\mathrm{Cu}$, we estimate $\chi_{\mathrm{diam}}=-1 \times 10^{-6}$ (units of $\chi$ are in $\mathrm{emu} / \mathrm{cc}$ ), which may be considered an upper limit because in both $\mathrm{Fe}$ and $\mathrm{Ni}$ the $d$ band is not completely filled, and it is known that the $3 d$ electrons are the major contributors to $\chi_{\text {diam }}$. Similarly $\chi_{s}$ is very small in $\mathrm{Ni}\left(\sim 0.9 \times 10^{-6}\right.$ in the free-electron approximation for 0.6 " $s$ " electrons) and in $\mathrm{Fe}\left(\sim 1 \times 10^{-6}\right.$ for one "s" electron).

A much larger contribution arises from $\chi_{\mathrm{vv}}$ for which estimates have been made for a number of metals including paramagnetic $\mathrm{Ni}$ (although none of these estimates considered ferromagnetically occupied bands).

\footnotetext{
7 N. Blum and L. Grodzins, Phys. Rev. 136, A133 (1964).

8 The source and absorber foils were $0.375 \mathrm{in}$. diam $\times 0.001$ in. thick and 0.625 in. diam $\times 0.0002$ in. thick, respectively.
}

They include $\mathrm{Ni}\left(1.1 \times 10^{-5}\right.$ and $\left.0.6 \times 10^{-5}\right)$, if a molecular field term is or is not included, respectively, $\mathrm{Cr}\left(2.0 \times 10^{-5}\right.$ and $\left.2.2 \times 10^{-5}\right), \mathrm{V}\left(2.3 \times 10^{-5}\right)$, and $\mathrm{Pt}$ $\left(0.3 \times 10^{-5}\right)$. We estimate that $\chi_{\mathrm{vv}} \simeq 1.1 \times 10^{-5}$ for $\mathrm{Ni}$ and $1.5 \times 10^{-5}$ for $\mathrm{Fe}$. By contrast, we note that the spin-wave contribution to the change in magnetization with field is completely negligible $\left(\chi \sim 10^{-8}\right)$ at low temperatures in the range of fields used here.

Estimates of $\chi_{d}$ can be made from band calculations using Eq. (1), but these are of necessity crude because in ferromagnets both the density-of-states term and the exchange term have approximately the same value. Note that while we have separated out $\chi_{\varepsilon}$ from $\chi_{d}$ in the above discussion the $N(\epsilon)$ terms from energy-band calculations contain combined $s$ and $d$ contributions. Since $\chi_{s}$ is very small, only a small error is made in using either procedure, but it does serve as a basis for making comparisons. For $\mathrm{Fe}$, Cornwell and Wohlfarth, ${ }^{10}$ using Wood's ${ }^{11}$ augmented plane-wave calculations, find $N\left(\epsilon_{F \uparrow}\right)=0.75$ and $N\left(\epsilon_{F \downarrow}\right)=0.35$ (states/atom $\cdot \mathrm{eV}$. spin) and a band splitting $\Delta E\left(=2 k \theta^{\prime} \zeta\right)=1.35 \mathrm{eV}$. This gives, by Eq. (1), $\chi_{d}=1.1 \times 10^{-5}$. Thus, the sum of $\chi_{d}$ and $\chi_{\mathrm{vv}}$ is consistent with our measured upper limit and supports the accepted view that both bands in Fe contain holes.

For $\mathrm{Ni}$, our present result $\chi<1.1 \times 10^{-5}$ indicates that the entire susceptibility could arise from $\chi_{\mathrm{vv}}$ within the estimated uncertainties in these contributions. Such a result is consistent with one full spin-band in $\mathrm{Ni}$, in agreement with previous expectations. ${ }^{12}$

Finally, Herring ${ }^{2}$ has used a simplified phenomenological description to estimate $\chi_{d}$. Using a difference in energy $\tilde{\Delta} E$ of $0.084 \mathrm{eV} /$ atom for $\mathrm{Fe}$, obtained from an analysis of magnetic specific-heat data, he finds $\chi_{d}=$ $2.7 \times 10^{-5}$. Applying this description to $\mathrm{Ni}$, and using ${ }^{2}$ $\Delta E=0.02 \mathrm{eV}$, one finds $\chi_{d} \approx 1 \times 10^{-5}$, which is larger than our estimated value and may be the result of a greater uncertainty in the estimate of $\tilde{\Delta} E$ from specific-heat data in this case.

Note added in proof. High-field susceptibility measurements for $\mathrm{Fe}$ and $\mathrm{Ni}$ have also been reported at this meeting by Herring et al. (p. 1340). Their values of $\chi$ are much larger than those reported here.

\section{ACKNOWLEDGMENTS}

We wish to thank A. C. Gossard and E. D. Thompson for helpful discussions.

\footnotetext{
${ }^{9}$ See for example, M. Shimizu, T. Takahashi, and A. Katsuki, J. Phys. Soc. Japan 18, 801 (1963). Because of space limitations, full references will be given in a forthcoming publication.

${ }^{10} \mathrm{~J}$. F. Cornwell and E. P. Wohlfarth, J. Phys. Soc. Japan 17, Suppl. B-1, 32 (1962).

i1 J. H. Wood, Phys. Rev. 126, 517 (1962).

12 See for example, J. C. Phillips, Phys. Rev. 104, 1263 (1956); ibid. 133, A1020 (1964); and E. D. Thompson, E. P. Wohlfarth, and A. C. Bryan, Proc. Phys. Soc. (London) 83, 59 (1964).
} 